(C)2018. American Geophysical Union. All Rights Reserved. Access to this work was provided by the University of Maryland, Baltimore County (UMBC)

ScholarWorks@UMBC digital repository on the Maryland Shared Open Access (MDSOAR) platform.

Please provide feedback Please support the ScholarWorks@UMBC repository by emailing scholarworks-group@umbc.edu and telling us what having access to this work means to you and why it's important to you. Thank you. 


\title{
Application of the Monte Carlo method in modeling dusty gas, dust in plasma, and energetic ions in planetary, magnetospheric, and heliospheric environments.
}

\author{
Valeriy Tenishev $^{1}$, Yinsi Shou ${ }^{1}$, Dmitry Borovikov ${ }^{1}$, Yuni Lee ${ }^{2,3}$, Nicolas \\ Fougere $^{1}$, Adam Michael $^{4}$, Michael R. Combi ${ }^{1}$ \\ ${ }^{1}$ Department of Climate and Space Sciences and Engineering, University of Michigan, MI 48109, USA \\ ${ }^{2}$ University of Maryland Baltimore County, 1000 Hilltop Cir, Baltimore, MD 21250, USA \\ ${ }^{3}$ NASA Goddard Space Flight Center, Greenbelt, MD, USA \\ ${ }^{4}$ Johns Hopkins University Applied Physics Laboratory, Laurel, MD 20723, USA
}

Key Points:

- Kinetic modeling is necessary for understanding various phenomena of planetary and space physics.

- AMPS is a versatile and well-tested code with a long track record of application to simulate various planetary and heliophysics phenomena.

- The paper demonstrates the modeling capabilities of AMPS by presenting several examples of the code's prior application.

Corresponding author: Valeriy Tenishev, vtenishe@umich.edu

This article has been accepted for publication and undergone full peer review but has not been through the copyediting, typesetting, pagination and proofreading process, which may lead to differences between this version and the Version of Record. Please cite this article as doi: 10.1029/2020JA028242.

This article is protected by copyright. All rights reserved. 


\begin{abstract}
Typical planetary and planetary satellite exospheres are in non-equilibrium conditions, which means that a distribution function that describes these environments is far from Maxwellian. It is even more true when considering transportation of energetic ions in planetary magnetospheres, making it necessary to solve the Boltzmann equation in order to capture kinetic effects when modeling evolution of the distribution function describing such environments. Among various numerical methods, the Monte Carlo approach is one of the most used one for solving kinetic equations. That is because of the relative simplicity of implementing and a high degree of flexibility in including new physical processes specific to a particular simulated environment. Adaptive Mesh Particle Simulator (AMPS) was developed as a general-purpose code for solving the Boltzmann equation in conditions typical for planetary and planetary satellite exospheres. Later, the code was generalized for modeling dusty gas, dust and plasma, and for simulating transportation of solar energetic particles and galactic cosmic rays in planetary magnetospheres. Here we present a brief overview of the design, list the implemented physics models, and outline the modeling capabilities of AMPS. The latter is supported by several examples of prior applications of the code.
\end{abstract}

\title{
1 Introduction
}

Analysis of the rarefied gas flows in conditions typical for space and planetary environments can be performed by solving the Boltzmann equation (Eq. 1) that describes the temporal evolution of a distribution function $f_{1}(\mathbf{x}, \mathbf{v})$ :

$$
\frac{\partial f_{1}}{\partial t}+\mathbf{v}_{1} \frac{\partial f_{1}}{\partial \mathbf{x}}+\dot{\mathbf{v}}_{1} \frac{\mathbf{F}}{m} \frac{\partial f_{1}}{\partial \mathbf{v}}=\frac{\delta f}{\delta t}
$$

where $\delta f / \delta t$ is the source term. That term accounting for collisions between gas molecules is the standard collision integral derived from kinetic theory

$$
\left(\frac{\delta f}{\delta t}\right)_{\text {coll }}=\int\left|\mathbf{v}_{2}-\mathbf{v}_{1}\right|\left(f_{1}\left(\mathbf{v}_{1}^{\prime}\right) f_{1}\left(\mathbf{v}_{2}^{\prime}\right)-f_{1}\left(\mathbf{v}_{1}\right) f_{1}\left(\mathbf{v}_{2}\right)\right) d \sigma d \mathbf{v}_{2} .
$$

Here, $\sigma$ is the total scattering cross section. The integral describes collisions, by which collision partners having velocities $\mathbf{v}_{1}^{\prime}$ and $\mathbf{v}_{2}^{\prime}$ get velocities $\mathbf{v}_{1}$ and $\mathbf{v}_{2}$ after the collision. The nature of the term $\delta f / \delta t$ allows one to include more complex physical processes in a simulation (such as chemical/photolytic reactions, energy exchange with internal degrees of freedom or interaction between multiple phases of the simulated environment).

A kinetic description becomes necessary when the collision frequency is not high enough to maintain the distribution function at the equilibrium state on the temporal and spatial scales of interest. In planetary and planetary satellite exospheres it is common that the gas density is low and the resulting collision frequency is insufficient to maintain the state of equilibrium in a simulated dusty gas flow because the temporal evolution of the distribution function in these environments is dominated by non-thermal processes (such as sputtering, sublimation, or photolytic reactions). The formal criterion for the need of a kinetic description is defined by the value of the Knudsen number

$$
K n=\frac{\lambda}{L},
$$

where $\lambda$ is the local mean free path, and $L$ is a characteristic length related to the dominant processes in the simulated environment. In a gas of hard spheres, the mean free path is $\lambda=1 /(\sqrt{2} n \sigma)$, where $n$ is the gas number density and $\sigma$ is the total collision cross-section.

The purpose of this paper is to introduce AMPS to a broad community. We do not intend this paper to be a manual for the code since the journal paper format is not ap- 
propriate for that purpose. Instead, the paper presents a range of the prior code's applications, so it could be used by the research community to decide whether the code is appropriate for their research.

AMPS is the result of almost two decades of active development. Its general-purpose modules contain about 130,000 lines of code written in C++. AMPS is not an open-source code in the strict sense of this definition, but it is available to the community in the source code as a component of the Space Weather Modelling Framework (SWMF). The SWMF is available for download at the Center for Space Environment Modeling (CSEM) of the University of Michigan (http://csem.engin.umich.edu/tools/swmf). AMPS is also available for runs on request through NASA's Community Coordinated Modeling Center (CCMC), where it can be used for tracing trajectories of charged particles in simulated magnetic fields (https://ccmc.gsfc.nasa.gov/models/modelinfo.php?model=AMPS). We still work on the preparation a comprehensive manual for the code. At this point, the most straightforward way of adapting AMPS to study a new problem is to start with multiple examples of the prior code's applications that also are a part of the code distribution. Those examples are simplified versions of simulations that we performed with AMPS previously. We not only keep them as the starting point for adapting the code for simulating a new environment, but also run them as a part of AMPS' test routine to check the code's consistency and ensure that any code modification has no negative effect on other simulations. These tests are performed nightly on several computers, local Mac and Linux workstations, and a supercomputer.

\subsection{Direct Simulation Methods}

The Direct Simulation Monte Carlo (DSMC) method introduced by Bird (1994) is one of the main numerical techniques used for modeling gas flows in the intermediate to high Knudsen number regimes. The most important aspect of the method is that modeling particle interaction via collisions is decoupled from that of their motion. The latter is achieved using a probabilistic simulation of relevant physical processes performed at the individual model particle level. The main advantage of such an approach is that it allows one to study the effect of a wide range of non-thermal physical processes such as chemical and photolytic reactions, relaxation of the internal degrees of freedom, or interaction between multiple phases in a dusty gas flow. One of the most important features of the DSMC method is that it does not require the formulation of the integro-differential equations that describe the evolution of a distribution function.

The basic idea behind the DSMC method is that the simulated system is represented by a large but finite number of model particles. The dynamics of those particles is governed by the same physical laws that govern the dynamics of real atoms and molecules in the simulated gas flow. Macroscopic parameters of the gas or plasma (such as bulk velocity, density, or temperature) are calculated by sampling microscopic properties of the model particles (such as location and velocity). Statistical weight, which is the number of the real molecules or atoms represented by a single model particle, is defined to relate the model particle population to the real gas flow.

Various numerical schemes of the DSMC method are based on the same assumptions as those that form the basis for the phenomenological derivation of the Boltzmann equation. The key concept that is in the foundation of all particle collision models is the concept of the collision frequency, $\nu$. Using a probability density, $\omega$, of a transition $\left(\mathbf{v}_{i}, \mathbf{v}_{j}\right) \rightarrow$ $\left(\mathbf{v}_{i}^{\prime}, \mathbf{v}_{j}^{\prime}\right)$ for a pair of the particles, the collision frequency is defined as

$$
\nu=\frac{n}{N} \sum_{i<j} \omega\left[\left(\mathbf{v}_{i}, \mathbf{v}_{j}\right) \rightarrow\left(\mathbf{v}_{i}^{\prime}, \mathbf{v}_{j}^{\prime}\right)\right] d^{3} \mathbf{v}_{i} \mathbf{v}_{j}=\frac{n}{N} \sum_{i<j} \sigma_{t}\left(g_{i j}\right) g_{i j},
$$

where $g_{i j}$ is the relative speed between particles of species $i$ and $j, \sigma_{t}\left(g_{i j}\right)$ is the total collision cross section that, in general, is a function of the relative particle velocity, $n$ is 
the number density, $N$ is the total number of the model particles in a cell, $(N-1) N / 2$ is a number of the collision pairs, $\mathbf{v}_{i, j}$ and $\mathbf{v}_{i, j}^{\prime}$ are velocities of the colliding particles before and after a collision (Ivanov et al., 1998). The Boltzmann collision integral describes the effect of the binary collisions. Following that, particle collision schemes developed within the frame of the DSMC method are limited to modeling the effect of the binary collisions.

Due to the statistical nature of the DSMC method, statistical noise is always present in any simulation. Noise filtering techniques that could be used to decrease the effect of this statistical noise are developed and discussed by, e.g., Boyd and Stark (1989), Kaplan and Oran (2002). For the purposes of the variance reduction and improving the statistic sample, a variable particle weight is often used too.

There are multiple applications of the DSMC method for modeling various planetary phenomena. Some of them are discussed by, e.g. Combi (1994), Tucker et al. (2013), and Prem et al. (2019).

\section{Adaptive Mesh Particle Generator (AMPS)}

One of the primary objectives that defined the design of AMPS, was to create a general-purpose code for solving the Boltzmann equation that could be applied to simulating various space environments at conditions that go beyond those specified at the stage of designing the code. The most straightforward approach to achieve this goal was separating the general-purpose core from the specifics of particular applications.

This resulted in a multi-layer structure of AMPS that consists of three major components: (1) a preprocessor of the user input file, (2) the general-purpose core containing the library of models of the physical process describing the dynamics of the rarefied gas flows, the functionality that supports execution of the code when run on massively parallel computers, and (3) a user module that contains models of physical processes and characterization of the initial and boundary conditions that are specific to a particular simulated environment. Therefore, AMPS is designed as a tool-box that a user can easily apply for building models suitable for various environments and conditions. Such a design allows the code to be indeed a general-purpose one by providing the capability to model various environments without any modification of the core. The library of physics models included in AMPS contains various models of particle collisions, internal degrees of freedom, and photochemical reactions, as summarized in Table 1. Impact vaporization, thermal desorption, photo-stimulated desorption, sputtering models were used in our modeling of the lunar exosphere. AMPS provides to a user a framework for implementing customized models of various physical processes. Among many, that includes providing a user with a framework for implementing customized models of impact vaporization, thermal desorption, photo-stimulated desorption, and sputtering that would fit the user-specific application needs. Table 2 summarizes environments simulated with AMPS. The unique feature of AMPS is that those user-defined functions need only be registered with the core in order to be used in a simulation. Hence, a user does not need to know the intimate details of how AMPS is organized. That was one of the guiding principles of the AMPS' design.

The other purpose of the core is the domain decomposition, load balancing, and memory management that are necessary for efficient computations on massively parallel computers. The domain decomposition procedure is based on splitting the Morton curve. As the criterion of such splitting, AMPS is capable of using the particle number, the actual computational time measured by the code during a course of a simulation, or a user-defined criterion for a domain decomposition.

AMPS employs adaptive Mesh Refinement (AMR). The mesh library is a part of AMPS' general-purpose code. An example of the mesh used in our exploratory study 

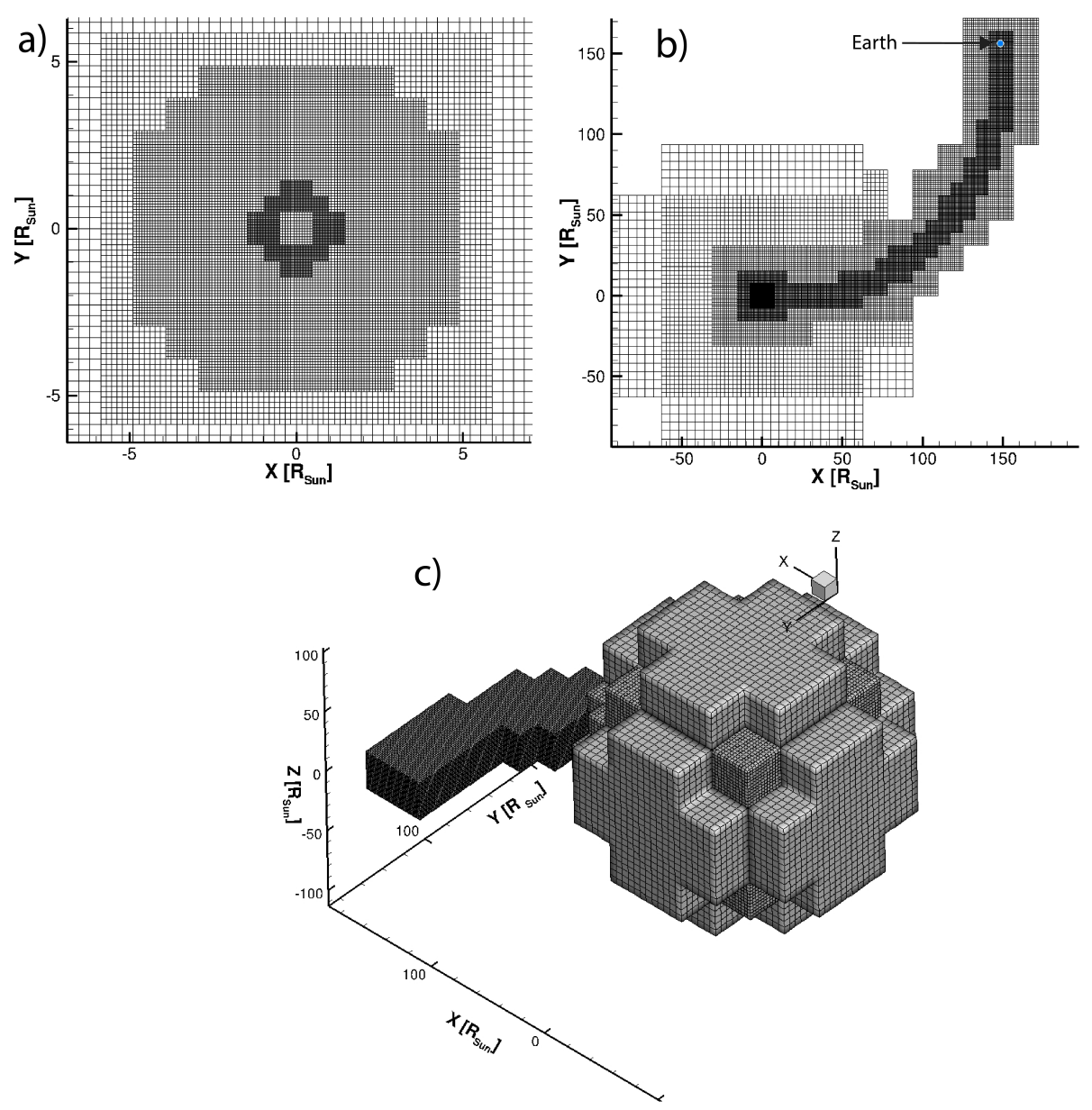

Figure 1. Example of a mesh used in simulations conducted using AMPS. This mesh was used in our exploratory study of the solar energetic particle propagation in the inner heliosphere. Panels a) and b) show the cut through the computational domain in the ecliptic plane. For that simulation, it is essential to both resolve the particle acceleration close to the Sun as well as their transport toward the Earth, which only can efficiently be achieved using the AMR approach. Panel a) shows a part of the domain that covers the region near the Sun. Panel b) shows the entire computational domain. The unique feature of AMPS is that it does not require a box-type domain. Solar energetic particles preferentially move along magnetic field lines. Therefore, there is no need to discretize the entire inner heliosphere. For saving computational resources, only the region near the Sun is covered completely. At larger heliocentric distances, the domain confines around the magnetic field line connecting the Sun to the Earth. Panel c) shows the shape of the computational domain.

of solar energetic particle propagation in the inner heliosphere is presented in Figure 1. The unique feature of the implemented approach is that the computational domain does not have to be rectangular. Instead, the domain can be designed such that it represents the simulated problem more efficiently. Figure 1 shows the computational domain that includes the Sun, the Earth, and a region surrounding the magnetic field lines connecting them. 
Modeling the planetary environment may require to simulate the interaction of the planet or other objects with the ambient gas. AMPS has the capability of modeling the gas/surface interaction with an arbitrarily complex surface. For that, AMPS employs the triangulated surface representation. In a simulation, the object is "cut" out of the computational domain using the cut-cell approach. An example of the surface that was used in one of our prior simulations conducted using AMPS is presented in Figure 2. Details of that modeling is discussed in Section 3.2. AMPS uses NAIF SPICE for calculating the relative positions and orientations of astronomical objects, spacecraft and pointing directions of the spacecraft mounted instruments.
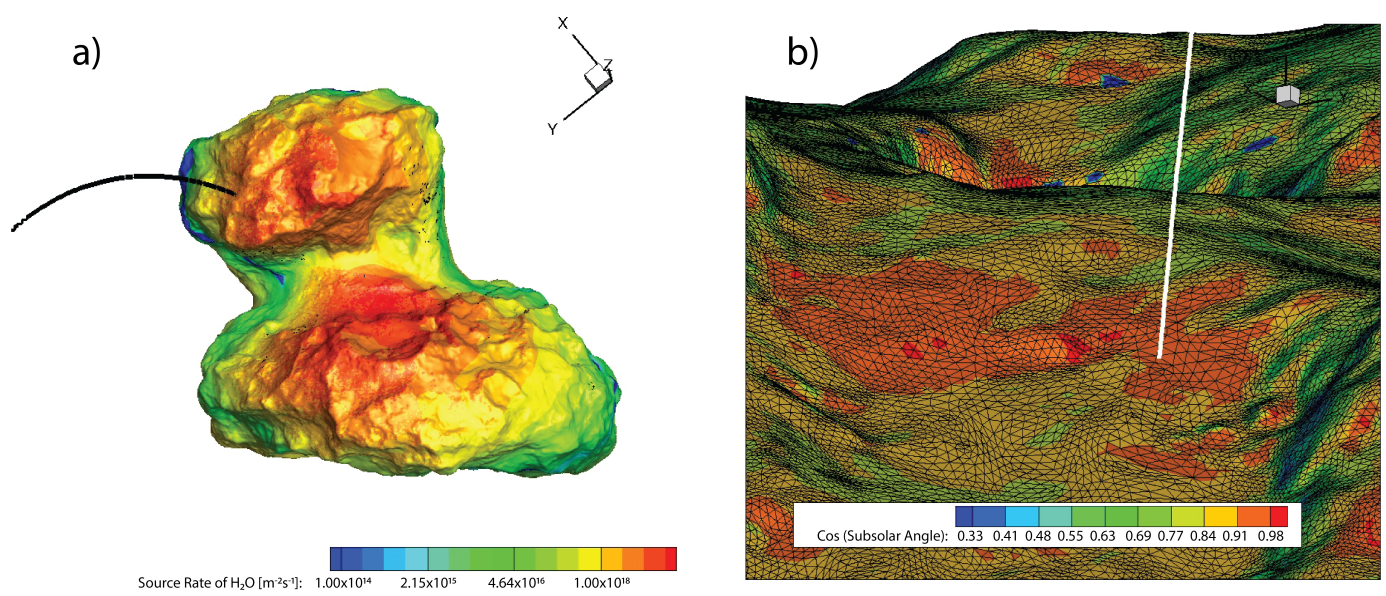

Figure 2. The figure illustrates the complexity of the shape used when simulating the coma of comet $67 \mathrm{P} /$ Churyumov-Gerasimenko discussed later in Section 3.2. Panel a) shows the entire nucleus and the distribution of the volatile source rate across the surface. The black line is the trajectory of Rosetta during the final landing to the comet's nucleus. The panel b) shows the surface triangulation of the nucleus shape that AMPS can use for representing the objects inside the domain. The color represents the value of the cosine of the subsolar angle at the time of the spacecraft touchdown at 11:19 a.m. GMT on 30 September 2016. The white line on panel is the trajectory of the spacecraft during the final landing phase. AMPS uses NAIF SPICE to calculate the relative positions and orientations of astronomical objects, spacecraft and spacecraft mounted instruments.

AMPS is capable of refining the mesh automatically. In the current implementation, AMPS was used only for modeling 3D flows. 2D could be achieved by limiting the third dimension in a 3D simulation. However, the capability of modeling in $1 \mathrm{D}$ and $2 \mathrm{D}$ was a part of the original design of AMPS. During the code development, only 3D was used, and hence, only development of the 3D modeling capabilities was progressed. However, because of the code's design, the dimension-related parts of the code are isolated and could be added in case needed.

Solving the Boltzmann equation, AMPS is valid in the entire range of collision regimes starting from a collisionless and to collision dominated. In the latter case, the computational cost of employing the DSMC method in a simulation could become prohibitively high. In that case, fluid approaches (Navier-Stokes or Euler) that impose the assumption of equilibrium on the gas distribution function become more computationally efficient (Bird, 1994). In general, kinetic effects become a factor affecting gas flow dynam- 


\begin{tabular}{ll}
\hline Section & Implemented models \\
\hline Trajectory integration & Boris algorithm, Second order leap frog integrator \\
Particle collision model & Non-time counter (NTC), Majorant Frequency (MF) \\
Internal degrees of freedom & Borgnakke and Larsen (LB) model \\
Type of the surfaces & $\begin{array}{l}\text { Sphere, Body of rotation, Generic triangulation of the } \\
\text { surface shape }\end{array}$ \\
Parallel implementation & Domain decomposition, MPI+OpenMP implementation \\
Real gas effects & Photolytic reaction model \\
Dust & Charging dust grains, Gas drag \\
\hline
\end{tabular}

Table 1. The lists of physics models implemented in AMPS.

ics at the Knudsen number $K n>0.01$. Though valid at smaller values of the Knudsen number, AMPS' application is optimal at $K n>0.01$.

\subsection{Modeling neutral gas flows}

Because modeling of all physical processes is done on the level of individual model particles, a realistic simulation of a dusty gas flow with the DSMC method requires employing an efficient physics model of the relevant processes. The numerical procedures and functions that simulate those processes would be executed multiple times during a single time step and applied to pairs or individual model particles. The underlying assumption of all physics models implemented within the DSMC method is that a characteristic time associated with a simulated physical process is much shorter than the typical time associated with the evolution of the simulated system as a whole. That allows one to treat such processes as a serious of instantaneous events that occur with a rate defined by the properties of the simulated environment.

\subsubsection{Collision Dynamics}

A number of collision models have been developed within the frame of the DSMC method. In simulations relevant to planetary applications of AMPS, the collision time during which particles can interact with each other is much shorter than that of the mean time between collisions. That makes it possible to introduce a probabilistic model that would describe the state of the collided particles after a collision based on their parameters before the collision.

All collision algorithms developed within the frame of the DSMC method share a similar criterion of acceptance of a collision, but differ in the way the number of the collisions is calculated. In the first class of the algorithms, the total number of particle collisions between the model particles that could happen in a computational cell during a single time step is evaluated at the beginning of the simulation procedure. In another class of the collision schemes, a collision frequency is assumed to be constant during a time step, and, therefore, the time intervals between following collision events are distributed according to Poisson distribution. The characteristic of all collision models developed within the DSMC method is conservation of energy and momentum on the level of the individual colliding particle pairs:

$$
\begin{gathered}
\mathbf{p}_{1}^{\prime}=m_{r} \mathbf{c}_{r}^{\prime}+\frac{m_{r}}{m_{2}}\left(\mathbf{p}_{1}+\mathbf{p}_{2}\right) \\
\mathbf{p}_{2}^{\prime}=-m_{r} \mathbf{c}_{r}^{\prime}+\frac{m_{r}}{m_{1}}\left(\mathbf{p}_{1}+\mathbf{p}_{2}\right)
\end{gathered}
$$




\begin{tabular}{|c|c|c|}
\hline Environment & Employed model components & References \\
\hline $\begin{array}{l}\text { Mars' exosphere } \\
\text { and corona }\end{array}$ & $\begin{array}{l}\text { Neutral particles, non-thermal popula- } \\
\text { tion, thermalization via collisions with } \\
\text { the background atmosphere }\end{array}$ & $\begin{array}{l}\text { Valeille et al., 2009a, } \\
\text { 2009b; Lee et al., 2014a, } \\
2014 b\end{array}$ \\
\hline $\begin{array}{l}\text { Exosphere of } \\
\text { the Moon }\end{array}$ & $\begin{array}{l}\text { Neutral particles, non-thermal pop- } \\
\text { ulation, the non-inertial frame of } \\
\text { reference that accounts for rotation } \\
\text { of the Moon, the orbital motion of } \\
\text { the Moon around the Earth, and the } \\
\text { orbital motion of the Earth around } \\
\text { the Sun; thermal accommodation } \\
\text { at the Moon's surface, surface ad- } \\
\text { sorption/desorption, volatile surface } \\
\text { reservoir, photoionization }\end{array}$ & Tenishev et al., 2013 \\
\hline $\begin{array}{l}\text { Coma of comet } \\
67 \mathrm{P} / \text { Churyumov- } \\
\text { Gerasimenko }\end{array}$ & $\begin{array}{l}\text { Neutral particles, non-thermal pop- } \\
\text { ulation, irregularly shaped nucleus, } \\
\text { photolytic reactions, internal degrees } \\
\text { of freedom, inter-particle collisions, } \\
\text { gas/surface interaction }\end{array}$ & $\begin{array}{l}\text { Tenishev et al., 2008; } \\
\text { Fougere et al., 2012, } \\
\text { 2014, 2016; Combi et al., } \\
2012,2020,\end{array}$ \\
\hline $\begin{array}{l}\text { Dust in a } \\
\text { comet's en- } \\
\text { vironment }\end{array}$ & $\begin{array}{l}\text { Concurrent modeling of dust and } \\
\text { gas, acceleration due to drag by the } \\
\text { ambient gas }\end{array}$ & $\begin{array}{l}\text { Tenishev et al. 2011, } \\
2016\end{array}$ \\
\hline $\begin{array}{l}\text { Hydrogen pop- } \\
\text { ulation in the } \\
\text { outer helio- } \\
\text { sphere }\end{array}$ & $\begin{array}{l}\text { Non-thermal population, interaction } \\
\text { of the neutral hydrogen population } \\
\text { with solar wind via charge exchange, } \\
\text { production of the energetic neutral } \\
\text { atoms (ENAs) via charge exchange } \\
\text { reactions }\end{array}$ & Kornbleuth et al., 2020 \\
\hline
\end{tabular}

Table 2. Summary of the prior applications of AMPS. 
Here, $\mathbf{p}_{1,2}$ and $\mathbf{p}_{1,2}^{\prime}$ are the momenta of colliding particles before and after collision, $m_{1,2}$ are masses of the individual particles, $m_{r}=m_{1} m_{2} /\left(m_{1}+m_{2}\right)$ is the reduced mass, and $\mathbf{c}_{r}^{\prime}$ is the relative velocity of the particles in the center of mass frame of reference.

Two particle collision models were implemented in AMPS and can be used in modeling gas flows when collisions are important.

Non-Time Counter scheme. The Non-Time Counter scheme (NTC) is one of the schemes that are based on evaluating the number of prospective collisions between the model particles in a cell and then selecting a colliding particle pair from the particles in the cell. The number of prospective collisions is evaluated before the randomly selected pairs are checked for a possible collision (Bird, 1994; Abe, 1993). The total number of the model particle pairs that are checked for collisions is

$$
\frac{1}{2} N(N-1) w \frac{\left[\sigma_{T}\left(c_{r}\right) c_{r}\right]_{\max }}{d_{m}} \Delta t
$$

Here, $N$ is the total number of the model particles in a cell, $w$ is a particle statistical weight, $d_{m}$ is the volume of a cell, $\Delta t$ is the time step and $\left[\sigma_{T}\left(c_{r}\right) c_{r}\right]_{\max }$ is the upper limit of the product of the total collision cross section, $\sigma_{T}\left(c_{r}\right)$, and the relative particle velocity $c_{r}$.

Pairs are randomly selected from the pool of particles populating a computational cell. The probability for a particle pair to participate in a collision is proportional to the product of the collision cross-section value and the relative speed of prospective collision partners. To account for that, each selected pair is accepted for modeling a collision event with a probability

$$
p=\frac{\sigma c_{r}}{\left[\sigma c_{r}\right]_{\max }} .
$$

Majorant frequency scheme. Majorant frequency scheme is the second particle collision model available in AMPS. Contrary to NTC, this approach uses an estimate of the collision frequency, and the time interval between successive collision events, $\tau$, is distributed according to the Poisson distribution $p(\tau) \sim \exp (-\nu \tau)$, where $\nu$ is the majorant frequency of collisions and is defined as

$$
\nu=\frac{1}{2} N(N-1) w \frac{\left[\sigma_{T}\left(c_{r}\right) c_{r}\right]_{\max }}{d_{m}} .
$$

A pair is selected for modeling a collision with the probability in Eq. 8. The collision frequency can also be defined for the entire computational domain

$$
\nu=\sum_{k=1}^{M} \nu_{k}=\sum_{k=1}^{M} \frac{1}{2} N_{k}\left(N_{k}-1\right) w \frac{\left[\sigma_{T}\left(c_{r}\right) c_{r}\right]_{\max }}{d_{m}},
$$

where $M$ is the number of cells. The cell where a next pair of particles is checked for a collision is determined with the probability $p=\nu_{k} / \nu$.

Using a collision frequency calculated for the entire computational domain is one of the techniques for reducing the statistical noise that is unavoidable in Monte Carlo simulations. Such techniques are especially important in large-scale simulations. When the number of the model particles per cell is limited, applying collision models on the cell-by-cell basis might result in the frequency of the particle collision events deviating from their theoretical value. To reduce the effect of the statistical noise, the collision event rate can be calculated not for a given cell, but the entire domain. That will significantly increase the collision scheme's accuracy because the number of simulated collisions will increase. The prospective collision is considered successful with probability in Eq. 8. Markelov and Ivanov (2000) suggested that 5-10 particle per cell is usually sufficient for gas flows where chemical reactions are not important. 


\subsubsection{Internal Energy Models}

Energy exchange between the translation and internal degrees of freedom of molecules may have an important effect on the structure of the rarefied gas flow. A Borgnakke-Larsen model is included in AMPS for simulating that effect. The detail of the Borgnakke-Larsen model is described by Borgnakke and Larsen (1975). The major assumption of the model is that the energy spectrum of internal modes is continuous, and the post-collision energy is sampled from the local Boltzmann distribution

$$
f(\epsilon) \propto \epsilon^{\zeta / 2-1} \exp \left(-\frac{\epsilon}{k T}\right),
$$

where $\zeta$ is number of degrees of freedom, and $\epsilon$ is the internal energy.

The probability that in a collision event, there will be energy exchange between internal and translation degrees of freedom is $p=1 / Z_{r, v}$, which is the fraction of inelastic collisions. Here, $Z_{r, v}$ are the rotational and vibration collision numbers, respectively. The generally accepted approximation is $Z_{r}=5$ (Gimelshein et al., 1998; Gimelshein et al., 1999).

Polyatomic molecules process several vibration modes. Each of the modes contributes to the total vibration energy and can participate in the energy exchange independently. The vast majority of the polyatomic molecules show a single vibration relaxation (Lambert, 1977), which means that only one vibration mode participates in the energy exchange during a collision. Following Gimelshein et al. (1999) vibration relaxation model implemented in AMPS prohibits multiple relaxations (such as vibration energy relaxation in both collision partners or two different modes of the same particles).

\subsubsection{Photolytic reactions}

The model of the photolytic reactions implemented in AMPS employs a speciesspecific and radiation-environment-specific life-time, $\tau_{0}$, to determine the probability that a model particle participates in such a reaction. The probability of the reaction to occur during time interval $\tau$ is expressed by

$$
p=1-e^{-\tau / \tau_{0}} .
$$

Photolytic reactions are often associated with excess energy released in e.g., photodissociation of a molecule. In AMPS, parameters of each reaction are stored in tables containing rates for each reaction channel, the list of the products, and the excess energies for each reaction channel. Appending those tables, a user can include new photolytic reactions in the simulation.

AMPS provides users with the capability for employing more sophisticated models of photolytic reactions. For that, a user needs to develop and register with the core two functions where the first one returns a lifetime of a particle. The second one simulates the appropriate transformation of the particle. AMPS would call those functions to determine the probability of a reaction to occur during a given time step and, in case a particle participated in that reaction, to simulate the outcome, respectively.

\subsection{Modeling Dust in Gas and Plasma Flows}

A wide range of practically important problems requires modeling of the multi-phase dusty gas and plasma flows. AMPS has the capability for modeling neutral and electrically charged dust. In the implemented model, the dust grains are assumed to be chemically inert and spherical (Burt \& Boyd, 2003; Benson et al., 2002; Morris et al., 2015). 
Motion of the dust drains is defined by the equation of motion

$$
\frac{4 \pi}{3} \rho_{d} a^{3} \frac{d \mathbf{v}_{d}}{d t}=\frac{1}{2} \pi a^{2} C_{D} \rho\left|\mathbf{u}-\mathbf{v}_{d}\right|\left(\mathbf{u}-\mathbf{v}_{d}\right)+\mathbf{F}_{d},
$$

where $\mathbf{F}_{d}$ is the macroscopic force acting upon on a dust grain, $\mathbf{v}_{d}$ is the velocity of the dust particle with radius $a$ and bulk density of $\rho_{d}$, and $C_{D}$ is the drag coefficient, and $\mathbf{u}$ and $\rho$ are the bulk velocity and the mass density of the ambient gas, respectively. In case when gravity has important effect on the dust grain trajectory the external force, $\mathbf{F}$ is

$$
\mathbf{F}=\frac{4 \pi}{3} \rho_{d} a^{3} \frac{G M}{r^{2}} \frac{r}{|r|},
$$

where $G$ is the gravitational constant, $M$ and $r$ are the mass and distance to the body (Tenishev et al., 2011).

\subsubsection{Charging of the dust grains}

In most space environments, dust particles are exposed to plasma and UV radiation and, consequently, carry electrostatic charges. Their motion is influenced by electric and magnetic fields in addition to gravity, drag, and radiation pressure from sunlight. The dynamics of small charged dust particles can be surprisingly complex, leading to levitation, rapid transport, energization and ejection, capture, and the formation of new planetary rings.

The dynamics of the electrical charge in plasma is described by the current balance equation $d Q / d t=\sum_{k} J_{k}$, where $J_{k}$ are the charging currents. In most space plasmas electron and ion collection currents and secondary and photoelectron emission currents dominate (Shafiq et al., 2011; Horányi et al., 2004). Models of the electron collection and ion collection currents, photo-electron current, and secondary electron current are implemented in AMPS following the description of these currents by Horányi (1996).

\subsection{Tracing charged particles in the background electromagnetic fields}

One of the AMPS' distinct features is the capability to track charged particles in the background electromagnetic field. The code allows a user to import the field information from a file, use the empirical model of the geomagnetic field T96 (Tsyganenko, 1995), or to receive simulated plasma and magnetic field data run-time through coupling from the global MHD code SWMF/BATSRUS (Tóth et al., 2012) as described in Section 2.4. AMPS can import .dat files produced by the Tecplot visualization package and import background plasma and field data saved by the SWMF/BATSRUS using the SWMF/BATL library.

A unique feature of the code is that it allows one to use precalculated time-dependent fields stored in a set of files. For that, AMPS replaces the fields loaded in memory runtime and applies a linear interpolation in time and space to determine the field value in an arbitrary location.

AMPS provides users with the capability for using customized data formats for storing all background data. The procedure for reading and parsing the files needs to be developed by the user. This procedure would read the data, and saves it in the appropriate location of the cell centers' state vector that is defined during initializing AMPS.

The Boris algorithm and the guiding center approach are implemented in AMPS for tracing charged particles. Both relativistic and non-relativistic versions of those are implemented. For particle tracking, the time step is determined according to the expected characteristic value of the particle velocity and the cells size. To resolve a particle gyration, the time step can be split into multiple sub-steps, such that the gyration is correctly simulated. When the gyroradius is much smaller than any of the characteristic scales 
of the simulated problem, gyration becomes unimportant. In that case, guiding center approximation is a better choice.

AMPS provides a user with capabilities to use a customized procedure for particle tracking. To use these capabilities, users need to register such a function with the core. This mechanism allows one to build a sophisticated particle moving procedure. For example, this mechanism would enable one to create an advanced particle mover that applies relativistic treatments for some species (e.g., electrons) and non-relativistic for others (e.g., protons) or switch between those depending on the particle energy.

AMPS also provides a user with the capabilities of visualizing trajectories of the individual particles. Particles, whose trajectories would be saved in a separate file, are selected according to a user-defined criterion that needs to be registered with the core.

\subsection{Coupling to the Space Weather Modeling Framework}

AMPS is a fully functioning component of the Space Weather Modeling Framework (SWMF) (Tóth et al., 2012), where it is coupled to the global MHD code BATSRUS. Two- and one-way AMPS/BATSRUS coupling has been implemented. One-way coupling was used previously for simulating sputtering the surface of Europa by heavy ions. In that simulations, BATSRUS was used to model Europa interacting with the Jovian magnetosphere. AMPS was used to trace heavy ions in the magnetic field received from the MHD simulation run-time via the coupling. A simplified version of that model serves now one of the nightly tests that we run to verify the consistency of both codes nightly.

We use the two-way AMPS/BATSRUS coupling in a study of the solar wind propagating in the outer heliosphere as a part of the "Solar-wind with Hydrogen Ion Exchange and Large-scale Dynamics" (SHIELD) model (Kornbleuth et al., 2020). In the outer heliosphere, the interaction of the neutral population and solar wind ions via charge-exchange has a significant effect on the solar wind propagation. In that study, we use AMPS for simulating the neutral species. Being coupled with BATSRUS, it receives parameters of the simulated solar wind plasma from BATSRUS, simulates the plasma interaction with the neutrals and then passes the source terms to BATSRUS.

Recently, much effort has been made to implement the Particle-In-Cell (PIC) capabilities in AMPS to enable the code to calculate the electric and magnetic field selfconsistently. AMPS employed the energy-conserving semi-implicit particle-in-cell method (Lapenta, 2017). The semi-implicit nature of the methods allows the code to employ a coarser grid with a larger time step comparing to that of explicit PIC methods. The essential property of such an approach is that when the periodic boundary conditions are used in a simulation, the total energy, i.e., the sum of wave energy and particle energy, in the computational domain, is conserved within the round-off error.

AMPS and BATSRUS could be used in a plasma simulation, concurrently. The domains where the codes are applied are defined as AMPS simulating plasma in the regions where the kinetic effect dominates, and BATSRUS is used elsewhere. In a realistic space plasma simulations, kinetic effects are significant only in a relatively small part of the domain. Therefore, a combined approach would enable global plasma modeling that otherwise would be prohibitively expensive. The coupling is conducted run-time with cadence determined by the codes' numerical stability and the characteristic time of the simulated physical processes (Daldorff et al., 2014). Unlike other particle-in-cell models, AMPS provides users with the capability of employing the PIC approach in irregularly shaped areas. These capabilities could find practical applications in, e.g., modeling Earth's magnetotail and bow shock region. The PIC extension of AMPS is already implemented and is currently in the testing stage. A publication describing the details of the updated code is under preparation. Then the new PIC capabilities of AMPS will be available to the community. 


\section{Application of AMPS to model space and planetary environments}

AMPS has a long successful track record of modeling various space environments. This section summarizes some applications and illustrates the potential of the code for modeling various physical phenomena.

\subsection{Sodium Exosphere of the Moon}

The example presented in this section illustrates application of AMPS to modeling the sodium component of the lunar exosphere (e.g., Tenishev et al., 2013). The dynamics of the lunar environment is controlled by a complex interaction of multiple physical processes (Mendillo, 2001; Stern, 1999). Molecular collisions in the lunar exosphere are negligible above the surface. As a result, the structure and dynamics of the exosphere are determined mainly by the source processes (e.g., sputtering, impact vaporization) and photoionization of the exospheric species.

With AMPS, we have studied the temporal and spatial variability of the sodium component of the lunar exosphere and tail. Simulations were performed starting from the lunar surface extending up to 400 lunar radii in the anti-sunward direction. The results of this study are illustrated in Figure 3 that shows a comparison of the simulated sodium brightness with the ground-based observations, and the effect of the Earth's gravity on the structure of the extended sodium tail. The most interesting feature present in the latter is the effect of so-called gravitational focusing, which is an enhancement of the sodium density in the lunar tail in the vicinity of the Earth caused by the Earth's gravity.

The specifics of the lunar environment have been described in the user module. In particular, sodium efficiently sticks to the surface of the Moon on the night-side, and it is released back into the exosphere on the day-side. We have performed this modeling in the non-inertial frame of reference where the $\mathrm{x}$-direction was pointing toward the Sun, and the y-directions was opposite to the Moon's velocity. The rate and the vector of the rotation were calculated in the user module using NAIF SPICE. The user module also calculated the centrifugal and centripetal accelerations.

\subsection{Dusty gas coma of comet 67P/Churyumov-Gerasimenko}

The tenuous cometary coma is a unique phenomenon in the solar system because of the small influence that gravity has on the gas escaping from the cometary nucleus. The main difficulty in modeling these environments is a rapid increase of the mean free path with distance from the comet. As a result, the flow regime can vary starting from a fluid continuum at the sub-solar point near the nucleus to a free flow at larger distances or on the night side (Tenishev et al., 2008). Hence, studying a cometary coma requires the use of a kinetic approach (Combi, 1996; Crifo et al., 2005; Skorov et al., 2016). We have used AMPS to investigate the coma of comet $67 \mathrm{P} / \mathrm{Churyumov-Gerasimenko} \mathrm{in} \mathrm{many}$ occurrences as illustrated in this section (Tenishev et al., 2008; Fougere et al., 2012; Fougere, 2014; Fougere et al., 2016a, 2016b; M. Combi et al., 2020). The results reviewed in this section were obtained using a comet nucleus model having about 100,000 triangular facets. The boundary conditions are solely based on local solar illumination taking into account self-shadowing from the nucleus.

The model results were compared with observations performed with ROSINA (Balsiger et al., 2007) and VIRTIS-H (Coradini et al., 1999) instruments onboard Rosetta, providing the first direct comparison between these measurements. The correlation between the model and the data was observed to be larger than 0.8 , clearly showing agreement of the model results with observations. Examples of the calculated number density in the coma, and dust brightness are presented in Figs. 4 and 5. 

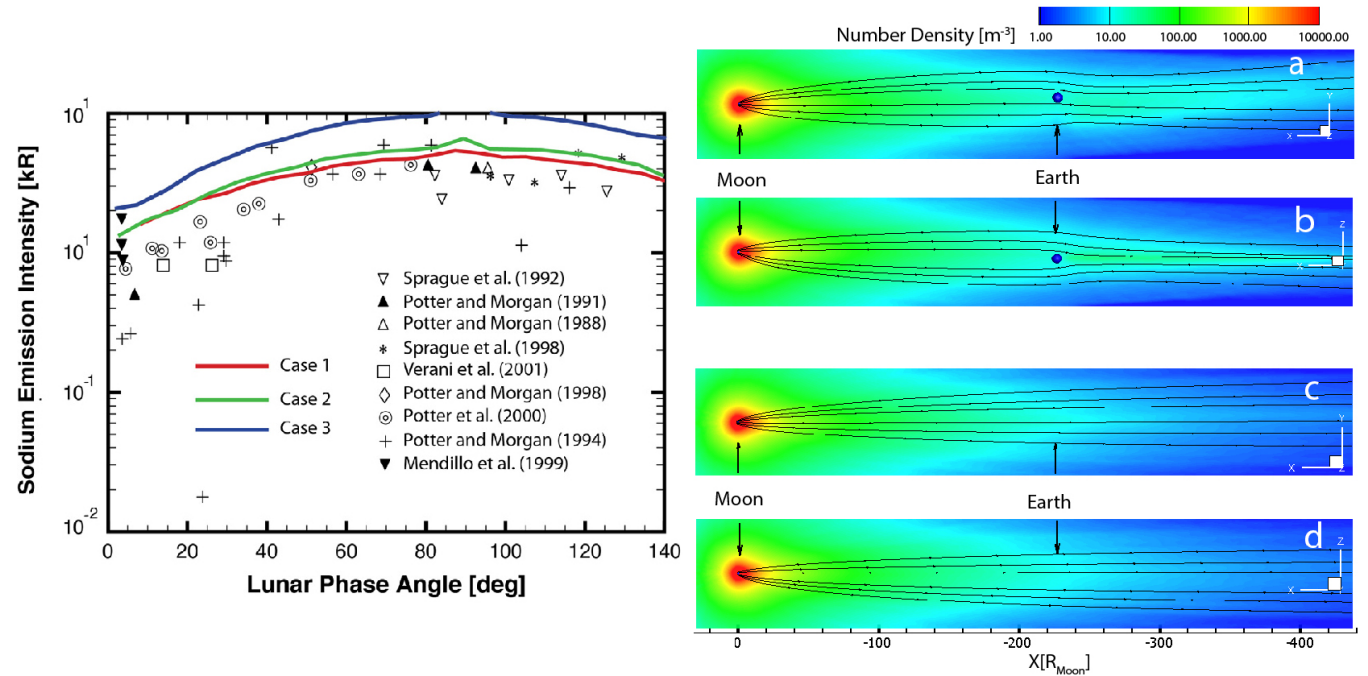

Figure 3. Left: Modeled brightness of the sodium D2 line at the lunar limb as it would be seen from the Earth. The plot presents a comparison of the model results with available groundbased observations (The statistics of observations is adapted from Sarantos et al. (2010)). Right: Simulated density distribution in the sodium tail. The coordinate frame is defined as follows: $x$ is directed to the Sun, $y$ is opposite to the velocity of the Moon, and $z=x \times y$ completes the right-handed system. The upper panel ( $\mathrm{a}$ and $\mathrm{b}$ ) shows the density distribution and streamlines in the tail as it passes the Earth. The lower panel (c and d) presents the same only when the Earth is far from the tail. The vertical arrows show the position of the Earth. It can be seen that Earth's gravity deflects the tail and causes a slight enhancement of the sodium density behind the Earth. In detail, the results are discussed in our paper by Tenishev et al. (2013).

The user module included several components defining 1) the volatile source rate at the irregularly shaped nucleus and the outcome of the gas/surface interaction processes, 2) specifying injection of the dust particles into the coma, and 3) setting the calculation of the column density and dust brightness integrals. For that, the user module called the NAIF SPICE library to calculate Rosetta's location and orientation relative to the comet's nucleus and the pointing directions of the spacecraft mounted instruments.

\subsection{Hot Oxygen Corona of the Mars' Atmosphere}

Observations of Mars suggest that during the early Solar System its atmosphere was much thicker and warmer than it is at the current epoch. Due to lack of an appreciable magnetic field, the Mars' upper atmosphere directly interacts with solar wind on a global scale, enhancing the atmospheric loss through various escape mechanisms. Heavy species, such as $\mathrm{O}$ and $\mathrm{C}$, have been known to escape the Martian atmosphere via nonthermal mechanisms, generating the hot atomic corona in its upper atmosphere. Modeling Mars' extended hot oxygen corona was one of the planetary applications of AMPS. Results of that study were detailed by e.g., Valeille et al. (2009a,2009b), and Lee et al. (2014a,2014b). A comparison of the modeled OI $130.4 \mathrm{~nm}$ brightness simulated using AMPS with the MAVEN/IUVS coronal limb scan data for orbit 236 is presented in Figure 6. The OI $1304 \mathrm{~nm}$ brightness is obtained by integrating the simulated exospheric $\mathrm{O}$ density along the line-of-sight of the instrument and adopting a g-factor of $4.74 \times 10^{-6} \mathrm{~s}^{-1}$. The IUVS observation in Figure 6 shows the two-component structure of the exospheric 

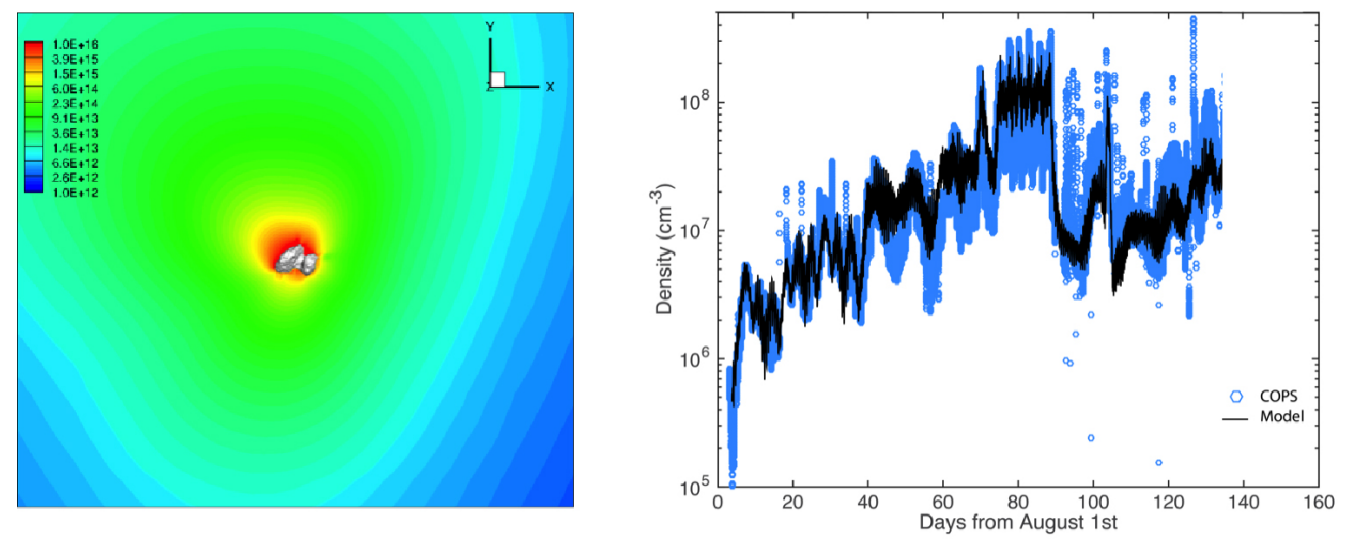

Figure 4. Left: Logarithm of the simulated $\mathrm{H}_{2} \mathrm{O}$ column density (in $\mathrm{m}^{-2}$ ) calculated at the location and orientation of the nucleus with respect to the Sun as on December 3rd, 2014, at 07:02 UT within the Rosetta/OSIRIS WAC FOV. Right: Comparison of the modeled $\mathrm{H}_{2} \mathrm{O}$ density with the density measured by ROSINA/COPS onboard Rosetta (in $\mathrm{cm}^{-3}$ ). It was shown in Bieler et al. (2015) that the model and the data have a correlation greater than 0.8 . This results are discussed in detail by Fougere et al. (2016a, 2016b).
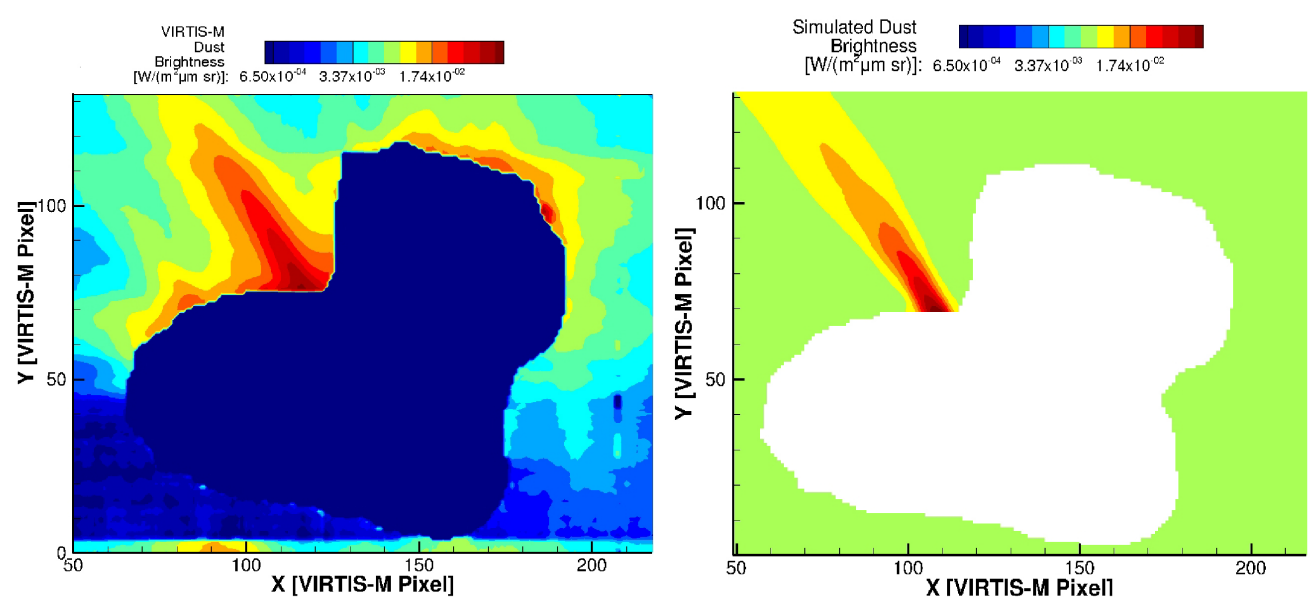

Figure 5. Comparison of the cometary dust brightness map observed by Rosetta VIRTIS-M [observation I1_00387442903 taken on 2015-04-12T07:14:00 (Migliorini et al., 2016)], with that from our kinetic modeling of gas and dust in the coma of comet $67 \mathrm{P} /$ ChuryumovGerasimenko. The observed brightness map is shown in the left panel, and the modeled one is shown in the right panel. X- and Y-axes represent the instrument pixel grid. In detail, the results are discussed in our paper by Tenishev et al. (2016).

O density profile composed of thermal and non-thermal populations with different scale heights. The non-thermal population with a relatively larger scale height dominates the total exospheric $\mathrm{O}$ density at altitudes above $600 \mathrm{~km}$. The non-thermal component of $\mathrm{O}$ is optically-thin, allowing the direct calculation of the $\mathrm{O} 130.4 \mathrm{~nm}$ brightness. As shown in Figure 6, the non-thermal O brightness predicted by AMPS reproduced the structure and magnitude of the observed OI $130.4 \mathrm{~nm}$ brightness at altitudes above $600 \mathrm{~km}$. 
In this study, the user module contained functions describing the non-thermal oxygen source in Mars' atmosphere. Accounting for collisions of the simulated "hot" oxygen with the background atmosphere is crucial. Therefore, the user module loaded the background atmosphere model files, calculated the energy-dependent collision cross-section, and defined the energy limit below which oxygen atoms are considered thermalized and, hence, are removed from the simulation.
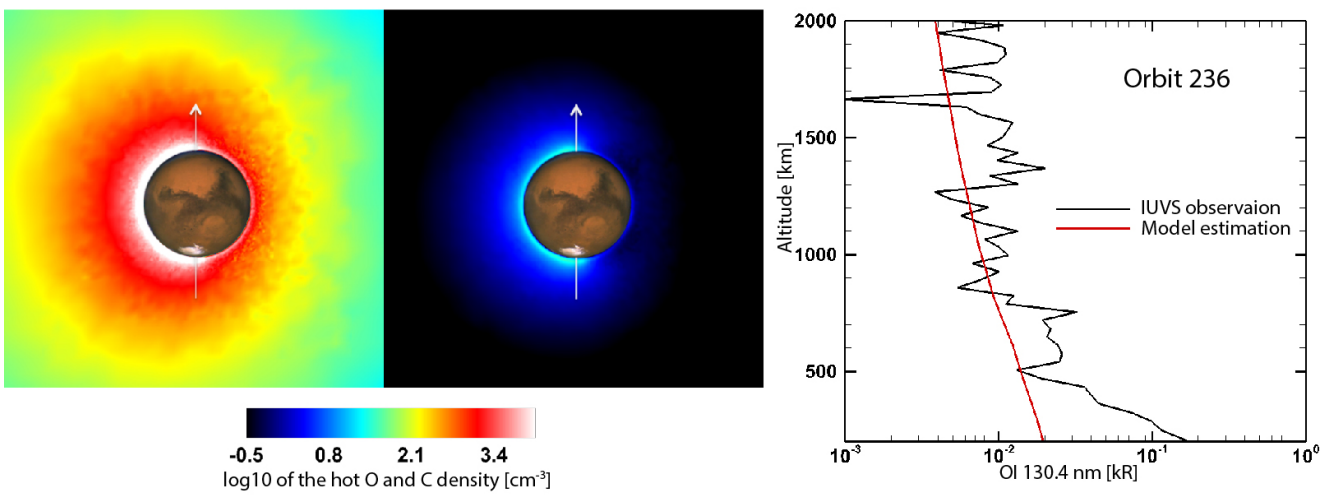

Figure 6. Left: The simulated Martian hot $\mathrm{O}$ corona (left) and hot $\mathrm{C}$ corona (right). The planes shown here are the Sun-Mars meridian plane with the Sun on the left. The color contour indicates the $\log 10$ of the hot $\mathrm{O}$ and $\mathrm{C}$ density $\left(\mathrm{cm}^{-3}\right)$. The arrow penetrating the planet is the axis of rotation, pointing the North pole. Right: A sample view of our model-data comparison (adapted from Lee et al., 2015a ). Since we model the hot O corona, our focus is on the altitude region where hot $\mathrm{O}$ dominates the exospheric $\mathrm{O}$. In this plot, hot $\mathrm{O}$ dominates above $670 \mathrm{~km}$. In detail, these results are discussed in our papers by Lee et al., (2014, 2015a,b).

\subsection{Energetic particles in the Earth's magnetosphere}

There are two populations of energetic particles that are important for assessing radiation hazard in geospace. According to the current paradigm, galactic cosmic rays (GCRs) are produced by diffusive shock acceleration in supernova remnants from which they diffuse to fill the whole galaxy (Blandford \& Eichler, 1987). The composition of GCRs is dominated by $\mathrm{H}^{+}$and $\mathrm{He}^{2+}$ (Simpson, 1983; Mewaldt, 1994). In order to be observed at Earth, these charged particles have to penetrate the electromagnetic fields of the heliosphere, i.e., the region of space around the Sun that extends to farther than $100 \mathrm{au}$ and is dominated by the solar-wind plasma and by the interplanetary magnetic field (IMF). On the other hand, Solar energetic particles (SEPs) are energetic particles ejected by the Sun in events that are associated with coronal mass ejections (CMEs) and solar flares (Reames, 1999).

We have used AMPS for simulating propagation of energetic particles in geospace under various geomagnetic conditions. Figure 7 presents results of modeling the propagation of GCRs in geospace under the conditions at the magnetopause taken from Badavi et al. (2011). For that we have performed global MHD modeling of the Earth's magnetosphere using SWMF/BATSRUS and assuming the following parameters of the solar wind: $v_{\mathrm{SW}}=400 \mathrm{~km} / \mathrm{s}, \mathrm{T}_{\mathrm{Sw}}=10^{5} \mathrm{~K}$, and $n_{\mathrm{SW}}=5 \mathrm{~cm}^{-3}$.

Rigidity cutoff is an essential characteristic of the geospace radiation environment. In SI system of units, it is defined as $p c / e$, where $p$ is the momentum of the particle, $c$ is the speed of light, and $e$ is the electric charge of the particle. Rigidity cutoff is a func- 

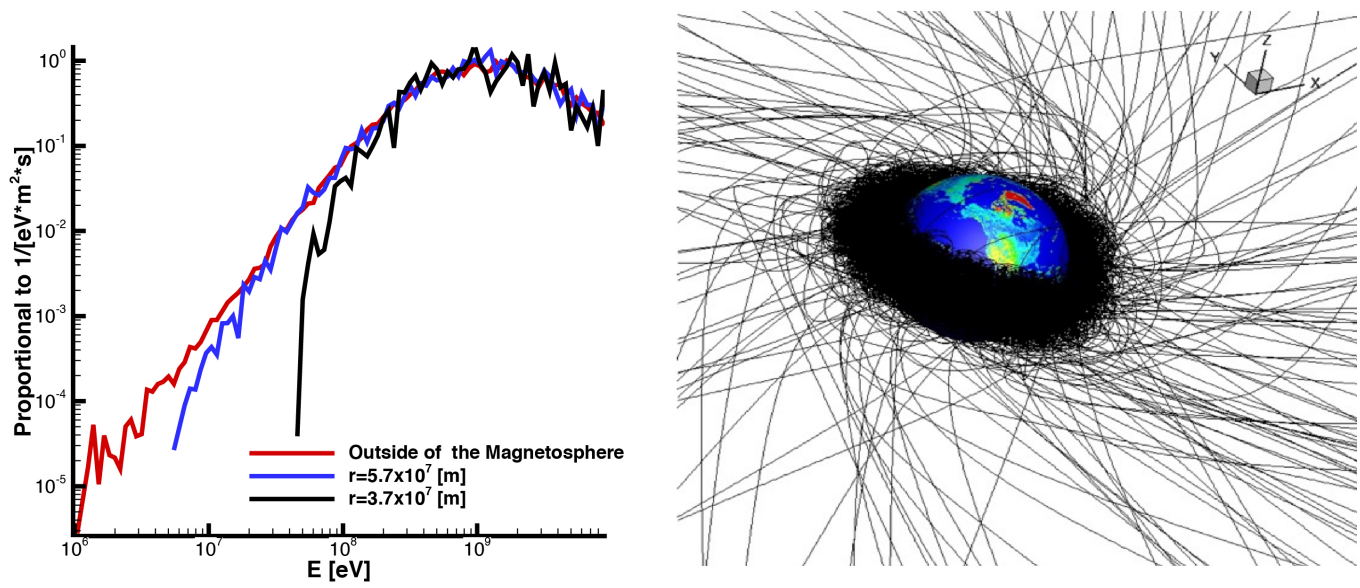

Figure 7. Left: An example of modeling of GCRs propagating in the Earth's magnetosphere. In this test we have determined variation of the GCRs energy-dependent flux at different geocentric distances $\left(r=3.7 \times 10^{7} \mathrm{~m}\right.$, and $\left.r=5.7 \times 10^{7} \mathrm{~m}\right)$, and compared those with GCRs flux outside of the magnetosphere. The latter was adopted from (Badavi et al., 2011). Our results indicate that GCR $\mathrm{H}^{+}$with kinetic energy below $100 \mathrm{MeV}$ are shielded by the Earth's magnetosphere, which is consistent with modeling by Badavi et al. (2011). Right: Forward modeling of the energetic particle transport in the Earth's magnetosphere. When flux and energy spectra have to be derived only in a limited number of locations backtracking of the energetic particle trajectory method is preferable because of smaller computation "cost." Here, backtracking means that the particle equation of motion is integrated backward in time. The figure illustrates the topology of the particle trajectories. The linear interpolation implemented in AMPS allowed us to conduct accurate particle tracing starting in the vicinity of the Earth out to the outer part of the magnetosphere.

tion of momentum and also implies the minimum energy level that a particle has to have to reach a particular location in geospace. Figure 8 shows a cutoff rigidity map calculated by AMPS. The map is calculated for the altitude of $500 \mathrm{~km}$ for quiet geomagnetic conditions $\left(p_{\mathrm{SW}}=2 \mathrm{nPa}, \mathrm{DST}=1 \mathrm{nT}, \mathrm{B}_{y}=-0.08 \mathrm{nT}\right.$, and $\left.\mathrm{B}_{z}=2 \mathrm{nT}\right)$. The right panel in Figure 8 shows a decrease of the cutoff rigidity during geomagnetic storm under conditions of the geomagnetic storm on March 17, $2015\left(p_{\mathrm{Sw}}=10 \mathrm{nPa}, \mathrm{DST}=-200\right.$ $\mathrm{nT}, \mathrm{B}_{y}=-7 \mathrm{nT}$, and $\left.\mathrm{B}_{z}=-10 \mathrm{nT}\right)$. The geomagnetic field derived from the T96 model (Tsyganenko, 1995) is used for the calculation. Using about 300 cores, it took about 1.5 hours for calculating a single cutoff rigidity map containing about $10^{5}$ points.

Energetic particles were traced backward in time generated at an altitude of 500 $\mathrm{km}$ uniformly around the Earth. Because of the domain decomposition design implemented in AMPS, the most efficient strategy for parallel calculations is to uniformly inject the model particles from the sphere where the cutoff rigidity is calculated and trace them back toward the computational domain boundary. That requires each particle to contain information about the location of its origin. The user module has reserved the necessary space in the particle state vector during AMPS' initialization. The user module injects particles and samples the cutoff rigidity when a particle reaches the computational domain boundary. 

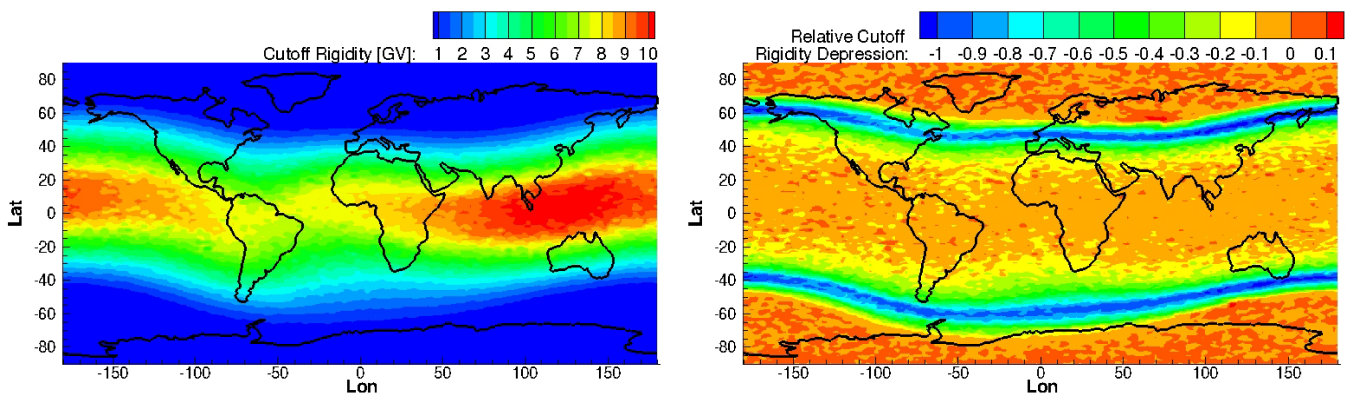

Figure 8. Example of applying AMPS for calculation rigidity cutoff. The map is calculated for an altitude of $500 \mathrm{~km}$. Left: Rigidity cutoff map calculated for quiet geomagnetic conditions. One clearly can see the location of the South-Atlantic Anomaly caused by the tilt of the Earth's magnetic dipole with respect to the planet's rotation axis. Right: Depression of the rigidity cutoff during a geomagnetic storm. The calculation was performed for conditions of the geomagnetic storm on March 17, 2015. One can see that the general rigidity cutoff patterns have changed mostly in the mid-latitude region.

\subsection{Hydrogen population in the outer heliosphere}
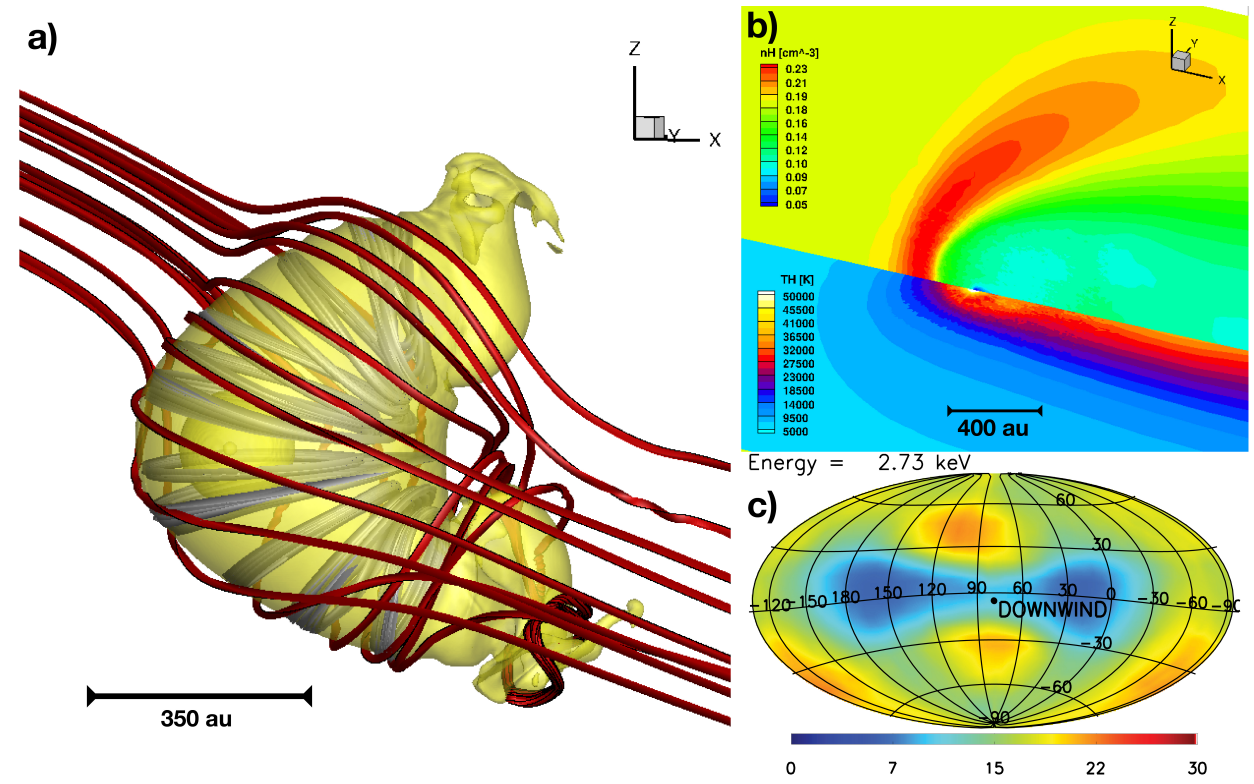

Figure 9. An example solution of the outer heliosphere produced by the two-way AMPS/BATSRUS coupled SHIELD model. a) is a side view of the heliosphere with the heliopause represented by the yellow isosurface. The red lines are interstellar magnetic field lines with solar magnetic field lines are in gray. The global neutral solution from AMPS is shown in b), with the neutral density in the meridional plane and the neutral temperature in the equatorial plane. Panel c) is adapted from Kornbleuth et al. 2020 and shows energetic neutral atom flux at $2.7 \mathrm{e} \mathrm{keV}$ as viewed from near Earth and centered on the downwind (tail) direction in units of $\left[\mathrm{cm}^{2} \mathrm{~s} \mathrm{sr} \mathrm{keV}\right]^{-1}$. These synthetic maps produced from the neutral solution of AMPS can be directly compared to IBEX and IMAP missions to probe the global shape of the heliosphere. 
The outer heliosphere is characterized by very large distances, as the solar wind expands into the interstellar medium. This forms a cavity on the order of 100 au surrounding the Sun and can affect the space environment in the wake of the heliosphere for thousands of au. The solar wind-interstellar medium interaction is another unique application where both fluid and kinetic processes are important. Neutral hydrogen is the dominant component of the interstellar medium and plays a crucial role in controlling the shape and size of the heliosphere (Baranov \& Malama, 1993). They stream through the heliosphere interacting with solar wind through resonant charge exchange. The Solarwind with Hydrogen Ion Exchange and Large-scale Dynamics (SHIELD) model is a global, self-consistent kinetic-MHD model of the outer heliosphere which utilizes AMPS to determine the evolution of the neutral atoms streaming through the heliosphere and the resulting impact of charge exchange on the plasma.

Figure 9 illustrates results of the SHIELD model and the application of AMPS to the outer heliosphere (Opher et al. 2015, Kornbleuth et al. 2020). This example illustrates the coupling of AMPS with the Outer Heliosphere $(\mathrm{OH})$ component of the SWMF. Here, in addition to specifying the boundary conditions, the user module needs to define the outcome of the interaction of species simulated with AMPS and those modeled with SWMF/BATSRUS. A simplified version of this model serves as a nightly test.

\subsection{Conclusion}

The primary purpose of the paper is to present a general-purpose kinetic particle code, AMPS, to the community, describe the basic principles of its design, list the implemented physical models, and give an overview of the range of possible applications. The advantage of AMPS as a modeling tool when simulating dusty gas, dusty plasma, and transportation of the energetic ions (e.g., ions of planetary origin, solar energetic particles, galactic cosmic rays in planetary magnetospheres) is the simplicity and flexibility of adapting the code to a particular environment.

The range of applications of the code is not limited by the functionality implemented in the general-purpose core of the code. The guiding principle of the code design was to create a tool that could be extended in the future to simulating various environments at conditions that go beyond the specifications at the stage of designing the code. The chosen design has allowed us to achieve this goal. The list of the prior applications of the code spans from planetary satellite and planetary exospheres and cometary comae up to modeling transportation of solar energetic particles and galactic cosmic rays in planetary magnetospheres.

AMPS was transitioned to the NASA's Community Coordinated Modeling Center (CCMC). As the code is now available to the community, we expect that AMPS will become a demanded modeling tool, and the list of its successful applications will multiply.

\section{Acknowledgments}

Support for this work was provided by grant 80NSSC17K0681 from the NASA Living with a Star Program and by NASA grant 18-DRIVE18_2-0029, Our Heliospheric Shield, 80NSSC20K0603. We also acknowledge the support by NASA's Community Coordinated Modeling Center during the transitioning of AMPS to the center. Data in Figures 1-2 and 7-9 is available in the University of Michigan Deep Blue Data archive (collections http://hdl.handle.net/2027.42/163321 and https://doi.org/10.7302/ek5p-ce08). Data in Figs. 3-6 is available through our prior publications by Tenishev et al. (2013, 2016), Fougere et al. (2016a, 2016b), and Lee et al., (2014a, 2015). 


\section{References}

Abe, T. (1993). Generalized scheme of the no-time-counter scheme for the DSMC in rarefied gas flow analysis. Computers 8 Fluids, 22(2-3), 253 - 257. Retrieved from http://www.sciencedirect.com/science/article/pii/ 004579309390057G doi: http://dx.doi.org/10.1016/0045-7930(93)90057-G

Badavi, F. F., Nealy, J. E., \& Wilson, J. W. (2011). The low earth orbit validation of a dynamic and anisotropic trapped radiation model through $\{$ ISS $\}$ measurements. Advances in Space Research, 48(8), 1441 - 1458. Retrieved from http://www. sciencedirect.com/science/article/pii/S0273117711004352 doi: http://dx.doi.org/10.1016/j.asr.2011.06.009

Balsiger, H., Altwegg, K., Bochsler, P., Eberhardt, P., Fischer, J., Jäckel, S. G. A., ... Wollnik, H. (2007). Rosina - rosetta orbiter spectrometer for ion and neutral analysis. Space Science Reviews, 128(1), 745-801.

Baranov, V. B., \& Malama, Y. G. (1993). Model of the solar wind interaction with the local interstellar medium numerical solution of self-consistent problem. Journal of Geophysical Research, 98(A9), 15157-15164.

Benson, C., Zhong, J., Gimelshein, S., Levin, D., \& Montaser, A. (2002). A comprehensive model for the simulation of aerosols at high gas temperatures. AIAA2002-3181.

Bieler, A., Altwegg, K., Balsiger, H., Berthelier, J.-J., Calmonte, U., Combi, M., ... Wurz, P. (2015). Comparison of 3d kinetic and hydrodynamic models to ROSINA-COPS measurements of the neutral coma of $67 \mathrm{P} / \mathrm{Churyumov-}$ Gerasimenko. Astronomy and Astrophysics, 583(A7).

Bird, G. (1994). Molecular gas dynamics and the direct simulation of gas flows. Oxford University Press.

Blandford, R., \& Eichler, D. (1987). Particle acceleration at astrophysical shocks: a theory of cosmic ray origin. Physics Reports, 154, 1-75.

Borgnakke, C., \& Larsen, P. S. (1975). Statistical collision model for Monte Carlo simulation of polyatomic gas mixture. Journal of Computational Physics, 18, 405-420.

Boyd, I. D., \& Stark, I. (1989). Statistical fluctuations in Monte Carlo calculations. In Rarefied gas dynamics: Theoretical and computational techniques (p. 245-257).

Burt, J., \& Boyd, I. (2003). A DSMC method for the modeling of two phase nonequilibrium flows. AIAA-2003-3496.

Combi, M. (1996). Time-dependent gas kinetics in tenuous planetary atmospheres: The cometary coma. Icarus, $123(1), 207-226$.

Combi, M., Shou, Y., Fougere, N., Tenishev, V., Altwegg, K., Rubin, M., .. Toth, G. (2020). The surface distributions of the production of the major volatile species, h2o, co2, co and o2, from the nucleus of comet $67 \mathrm{p} /$ churyumov- gerasimenko throughout the rosetta mission as measured by the rosina double focusing mass spectrometer. Icarus, 335.

Combi, M. R. (1994). The fragmentation of dust in the innermost comae of comets: Possible evidence from ground-based images. Astronomical Journal, 108(1), 304-312.

Combi, M. R., Tenishev, V. M., Rubin, M., Fougere, N., \& Gombosi, T. I. (2012). Narrow dust jets in a diffuse gas coma: A natural product of small active regions on comets. Astrophysical Journal, $749(1), 29$.

Coradini, A., Capaccioni, F., Drossart, P., Semery, A., Arnold, G., \& Schade, U. (1999). Virtis: The imaging spectrometer of the rosetta mission. $A d-$ vances in Space Research, 24(9), 1095 - $1104 . \quad$ Retrieved from http:// Www.sciencedirect.com/science/article/pii/S0273117799802038 doi: https://doi.org/10.1016/S0273-1177(99)80203-8

Crifo, J., Loukianov, G. A., Rodionov, A. V., \& Zakharov, V. V. $\quad$ (2005). Direct Monte Carlo and multifluid modeling of the circumnuclear dust coma. Spheri- 
cal grain dynamics revisited. Icarus, 176(1), 192-219.

Daldorff, L. K., Tóth, G., Gombosi, T. I., Lapenta, G., Amaya, J., Markidis, S., \& Brackbill, J. U. (2014). Two-way coupling of a global hall magnetohydrodynamics model with a local implicit particle-in-cell model. Journal of Computational Physics, 268, 236 - 254.

Fougere, N. (2014). The complex outgassing of comets and the resulting coma, a direct simulation monte-carlo approach (Unpublished doctoral dissertation). University of Michigan.

Fougere, N., Altwegg, K., Berthelier, J.-J., Bieler, A., Bockelée-Morvan, D., Calmonte, U., ... Tzou, C.-Y. (2016a). Direct simulation monte carlo modelling of the major species in the coma of comet $67 \mathrm{p} /$ churyumov-gerasimenko. Monthly Notices of the Royal Astronomical Society, 462(Suppl 1), S156-S169.

Fougere, N., Altwegg, K., Berthelier, J.-J., Bieler, A., Bockelée-Morvan, D., Calmonte, U., ... Tzou, C.-Y. (2016b). Three-dimensional direct simulation Monte-Carlo modeling of the coma of comet $67 \mathrm{P} /$ Churyumov-Gerasimenko observed by the VIRTIS and ROSINA instruments on board Rosetta. Astronomy and Astrophysics, 588, A134.

Fougere, N., Combi, M. R., Tenishev, V., Rubin, M., Bonev, B. P., \& Mumma, M. J. (2012). Understanding measured water rotational temperatures and column densities in the very innermost coma of comet $73 \mathrm{P} /$ Schwassmann-Wachmann 3 B. Icarus, 221(1), 174-185.

Gimelshein, S. F., Ivanov, M. S., \& Boyd, I. D. (1999). Modeling of internal energy transfer of polyatomic molecules in rarefied plume flows. AIAA-1999-738.

Gimelshein, S. F., Ivanov, M. S., Markelov, G. N., \& Gorbachev, Y. E. (1998). Statistical simulation of nonequilibrium rarefied flows with quasiclassical vibrational energy transfer models. Journal of Thermophysics and Heat Transfer, 12(4), 489-495.

Horányi, M. (1996). Charged dust dynamics in the solar system. Annual review of astronomy and astrophysics, 34, 383-418.

Horányi, M., Hartquist, T. W., Havnes, O., Mendis, D. A., \& Morfill, G. E. (2004). Dusty plasma effects in Saturn's magnetosphere. Reviews of Geophysic, 42, RG4002.

Ivanov, M. S., Gimelshein, S. F., \& Markelov, G. N. (1998). Statistical simulation of the transition between regular and mach reflection in steady flows. Computers and Mathematics with Applications, 35(1-2), 113-125.

Kaplan, C. R., \& Oran, E. S. (2002). Nonlinear filtering of statistical noise in DSMC solutions. AIAA-2002-211.

Kornbleuth, M., Opher, M., Michael, A. T., Sokol, J. M., Toth, G., Tenishev, V., \& Drake, J. F. (2020). The confinement of the heliosheath plasma by the solar magnetic field as revealed by energetic neutral atom simulations. arXiv:2005.06643 [astro-ph.SR].

Lambert, J. D. (1977). Vibrational and rotational relaxation in gases. Clarendon Press.

Lapenta, G. (2017). Exactly energy conserving semi-implicit particle in cell formulation. Journal of Computational Physics, 334, 349-366.

Lee, Y., Combi, M. R., Tenishev, V., \& Bougher, S. W. (2014a). Hot carbon corona in mars' upper thermosphere and exosphere: 1. mechanisms and structure of the hot corona for low solar activity at equinox. Journal of Geophysical Research: Planets, 119(5), 905-924.

Lee, Y., Combi, M. R., Tenishev, V., \& Bougher, S. W. ～(2014b). Hot carbon corona in mars' upper thermosphere and exosphere: 2. solar cycle and seasonal variability. Journal of Geophysical Research (Planets), DOI: 10.1002/2014JE004669, 2487-2509.

Lee, Y., Combi, M. R., Tenishev, V., Bougher, S. W., Deighan, J., Schneider, N. M., ... Jakosky, B. M. (2015). A comparison of 3-d model predictions of mars' 
oxygen corona with early maven iuvs observations.

Geophysical Research Letters, 42, 9015-9022.

Lee, Y., Combi, M. R., Tenishev, V., Bougher, S. W., \& Lillis, R. J. (2015). Hot oxygen corona at mars and the photochemical escape of oxygen - improved description of the thermosphere, ionosphere and exosphere. Journal of Geophysical Research (Planets), 120.

Markelov, G. N., \& Ivanov, M. S. (2000). Kinetic analysis of hypersonic laminar separated flows for hollow cylinder flare configurations. AIAA-2000-2223.

Mendillo, M. (2001). The atmosphere of the Moon. Earth, Moon and Planets, 85$86,271-277$.

Mendillo, M., Baumgardner, J., \& Wilson, J. (1999). Observational test for the solar wind sputtering origin of the Moon's extended sodium atmosphere. Icarus, 137(1), 13-23.

Mewaldt, R. A. (1994, October). Galactic cosmic ray composition and energy spectra. Advances in Space Research, 14, 737-747. doi: 10.1016/0273-1177(94) 90536-3

Migliorini, A., Piccioni, G., Capaccioni, F., Filacchione, G., Bockelée-Morvan, D., Erard, S., ... Fink, U. (2016). Water and carbon dioxide distribution in the $67 \mathrm{P} /$ Churyumov-Gerasimenko coma from VIRTIS-M infrared observations. Astron. Astrophys, 589, A45.

Morris, A. B., Goldstein, D. B., Varghese, P. L., \& Trafton, L. M. (2015). Approach for modeling rocket plume impingement and dust dispersal on the moon. Journal of Spacecraft and Rockets, 1-13. doi: 10.2514/1.A33058

Opher, M., Drake, J. F., Zieger, B., \& Gombosi, T. I. (2015). Magnetized jets driven by the sun: the structure of the heliosphere revisited. The Astrophysical Journal Letters, $800(2)$, L28.

Potter, A., \& Morgan, T. (1991). Observations of the lunar sodium exosphere. Geophysical Research Letters, 18(11), 2089-2092.

Potter, A. E., Killen, R. M., \& Morgan, T. H. (2000). V Variation of lunar sodium during passage of the Moon through the Earth's magnetotail. Journal of Geophysical Research, 105(E6), 15,073-15,084.

Potter, A. E., \& Morgan, T. H. (1988). Discovery of sodium and potassium vapor in the atmosphere of the Moon. Science, 241, 675-680.

Potter, A. E., \& Morgan, T. H. (1994). Variation of lunar sodium emission intensity with phase angle. Geophysical Research Letters, 21(21), 2263-2266.

Potter, A. E., \& Morgan, T. H. (1998). Coronagraphic observations of the lunar sodium exosphere near the lunar surface. Journal of Geophysical Research, 103 (E4), 8581-8586.

Prem, P., Goldstein, D., Varghese, P., \& Trafton, L. (2019). Coupled dsmc-monte carlo radiative transfer modeling of gas dynamics in a transient impactgenerated lunar atmosphere. Icarus, 326, 88-104.

Reames, D. (1999). Particle acceleration at the sun and in the heliosphere. Space Science Reviews, 90, 413-491.

Sarantos, M., Killen, R. M., Surjalal Sharma, A., \& Slavin, J. A. (2010). Sources of sodium in the lunar exosphere: Modeling using ground-based observations of sodium emission and spacecraft data of the plasma. Icarus, 205(2), 364-374.

Shafiq, M., Wahlund, J.-E., Morooka, M. W., Kurth, W. S., \& Farrell, W. M. (2011). Characteristics of the dust-plasma interaction near Enceladus' south pole. Planetary and Space Science, 59(1), 17-25.

Simpson, J. A. (1983). Elemental and Isotopic Composition of the Galactic Cosmic Rays. Annual Review of Nuclear and Particle Science, 33, 323-382. doi: 10 .1146 /annurev.ns.33.120183.001543

Skorov, Y., Reshetnyk, V., Lacerda, P., Hartogh, P., \& Blum, J. (2016). Acceleration of cometary dust near the nucleus: application to $67 \mathrm{p} /$ churyumovgerasimenko. Monthly Notices of the Royal Astronomical Society, 461, 3410- 

(1992). The sodium and potassium atmosphere of the Moon and its interaction with the surface. Icarus, 96(1), 27-42.

Stern, S. A. (1999). The lunar atmosphere: History, status, current problems, and context. Reviews of Geophysics, 37(4), 453-491.

Tenishev, V., Combi, M., \& Davidsson, B. (2008). A global kinetic model for cometary comae. The evolution of the coma of the Rosetta target comet Churyumov-Gerasimenko throughout the mission. Astrophysical Journal, 685, 659-677.

Tenishev, V., Combi, M. R., \& Rubin, M. (2011). Numerical simulation of dust in a cometary coma: Application to comet $67 \mathrm{P} /$ Churyumov-Gerasimenko. Astrophysical Journal, 732(2).

Tenishev, V., Fougere, N., Borovikov, D., Combi, M. R., Bieler, A., Hansen, K. C., ... Fink, U. (2016). Analysis of the dust jet imaged by Rosetta VIRTIS-M in the coma of comet 67P/Churyumov-Gerasimenko on April 12, 2015. Monthly Notices of the Royal Astronomical Society, doi: 10.1093/mnras/stw2793. doi: $10.1093 / \mathrm{mnras} / \mathrm{stw} 2793$

Tenishev, V., Rubin, M., Tucker, O. J., Combi, M. R., \& Sarantos, M. (2013). Kinetic modeling of sodium in the lunar exosphere. Icarus, 226 (2), 1538-1549.

Tóth, G., van der Holst, B., Sokolov, I. V., de Zeeuw, D. L., Gombosi, T. I., Fang, F., ... Opher, M. (2012). Adaptive numerical algorithms in space weather modeling. Journal of Computational Physics, 231(3), 870-903.

Tsyganenko, N. A. (1995). Modeling the Earth's magnetospheric magnetic field confined within a realistic magnetopause. Journal of Geophysical Research, 100, A4.

Tucker, O. J., Johnson, R. E., Deighan, J. I., \& Volkov, A. N. (2013). Diffusion and thermal escape of $\mathrm{H}_{2}$ from Titan's atmosphere Monte Carlo simulations. Icarus, 222(1), 149-158.

Valeille, A., Combi, M. R., Bougher, S. W., Tenishev, V., \& Nagy, A. F. Three-dimensional study of Mars upper thermosphere/ionosphere and hot oxygen corona: 2. solar cycle, seasonal variations, and evolution over history. Journal of Geophysical Research, 114, E11006.

Valeille, A., Tenishev, V., Bougher, S. W., Combi, M. R., \& Nagy, A. F. Three-dimensional study of Mars upper thermosphere/ionosphere and hot oxygen corona: 1. general description and results at equinox for solar low conditions. Journal of Geophysical Research, 114, E11005.

Verani, S., Barbieri, C., Benn, C. R., Cremonese, G., \& Mendillo, M. (2001). The 1999 Quadrantids and the lunar Na atmosphere. Monthly Notices of the Royal Astronomical Society, 327(1), 244-248. 